\title{
Zero-shot Generalization in Dialog State Tracking through Generative Question Answering
}

\author{
Shuyang Li, ${ }^{1,2}$ Jin Cao, ${ }^{1}$ Mukund Sridhar, ${ }^{1}$ Henghui Zhu, ${ }^{3}$ Shang-Wen Li, ${ }^{3}$ \\ Wael Hamza, ${ }^{1}$ Julian McAuley ${ }^{2}$ \\ ${ }^{1}$ Amazon Alexa AI, ${ }^{2}$ UC San Diego, ${ }^{3}$ AWS AI \\ $\{$ shl008, jmcauley\}eng.ucsd.edu \\ \{jincao, harakere, henghui, shangwel, waelhamz\}@amazon.com
}

\begin{abstract}
Dialog State Tracking (DST), an integral part of modern dialog systems, aims to track user preferences and constraints (slots) in taskoriented dialogs. In real-world settings with constantly changing services, DST systems must generalize to new domains and unseen slot types. Existing methods for DST do not generalize well to new slot names and many require known ontologies of slot types and values for inference. We introduce a novel ontology-free framework that supports natural language queries for unseen constraints and slots in multi-domain task-oriented dialogs. Our approach is based on generative questionanswering using a conditional language model pre-trained on substantive English sentences. Our model improves joint goal accuracy in zero-shot domain adaptation settings by up to 9\% (absolute) over the previous state-of-theart on the MultiWOZ 2.1 dataset.
\end{abstract}

\section{Introduction}

Dialog agents are gaining increasing prominence in daily life. These systems aim to assist users via natural language conversations, taking the form of digital assistants who help accomplish everyday tasks by interfacing with connected devices and services. A key component to understanding and enabling these task-oriented dialogs is Dialog State Tracking (DST): extracting user intent and goals from conversations via filling in belief slots (Lemon et al., 2006; Wang and Lemon, 2013). Assistive and recommendation use-cases for dialog agents in production settings are particularly challenging due to constantly changing services and applications with which they interface.

Traditional DST systems have achieved high accuracy when presented with a known ontology of slot types and valid values (Chen et al., 2020). In a real-world setting, however, a DST model must

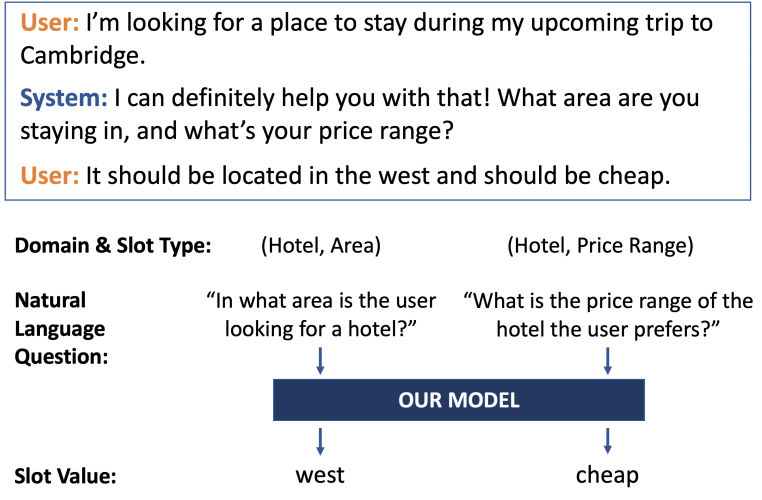

Figure 1: Based on a dialog history, a natural language questions are provided to our model to query a user's requirements and preferences (dialog state).

generalize to new slot values (e.g. new entities that are not present at training time) and new slot types (e.g. requirements regarding a new application). Recent work has sought to address these issues by posing DST as a reading comprehension or question answering (QA) task (Gao et al., 2019)—such models predict each slot value independently at any given turn and can theoretically be queried for new slots at inference time.

Some approaches toward DST as QA learn embedding vectors for each slot and/or domain word (Wu et al., 2019), but this is not robust to unseen slots whose specific names (e.g. 'Internet Access') may be totally unlike those in the training set. Gao et al. (2020) attempt to remedy this by posing a natural language question for each slot, but their hybrid span-extraction and classification-based system nonetheless requires access to the full ontology for unknown domains. We present an ontologyfree model using natural language questions to represent slots that builds on conditional language modeling techniques - taking advantage of the rise of powerful generative language models (Radford et al., 2019)—-to tackle DST as a generative QA 
task. Our model can generalize to unseen domains, slot types, and values, and allows developers to query for arbitrary user requirements via simple questions. To summarize our main contributions:

- We propose an ontology-free conditional language modeling framework for dialog state tracking via generative question answering, achieving state-of-the-art performance in zeroshot domain adaptation settings for DST on MultiWOZ 2.1 (Eric et al., 2020) across all domains with average per-domain gains of 5.9\% joint accuracy over previous best methods;

- We demonstrate performance competitive with state-of-the-art methods in a fully supervised setting;

- We show that our approach can be easily adapted to predict slot carry-over and transfer knowledge from a larger, more diverse dataset (Kim et al., 2019), improving zero-shot DST performance across all domains to $11 \%$ joint accuracy over the state-of-the-art.

\section{Approach}

We follow Gao et al. (2019) in treating Dialog State Tracking as a reading comprehension problem: at each turn of dialog, our model reads the dialog history and answers a fixed set of queries about user requirements and preferences (slots), with predictions aggregated to form the belief state. In our framework (Figure 1), we query for a given slot (e.g. Hotel Price Range) by asking a natural language question (Gao et al., 2020) - "What is the price range of the hotel the user prefers?". As our model's predictive ability is based on its general understanding of language and task-oriented conversation, we support zero-shot inference without the need to re-train the model or extend a formal ontology. For example, if a model has not been trained on data from the hotel domain, when presented with a hotel booking conversation we may nonetheless ask it a question like "In what area is the user looking for a hotel?" and received a prediction for that unseen requirement (Hotel Area).

While we conduct our experiments on Englishlanguage DST datasets, our approach is applicable to state tracking in any language, provided a conversation history is available.

Problem Statement We consider a conversation with $T$ turns of user $u_{t}$ and system utterances $y_{t}$ :

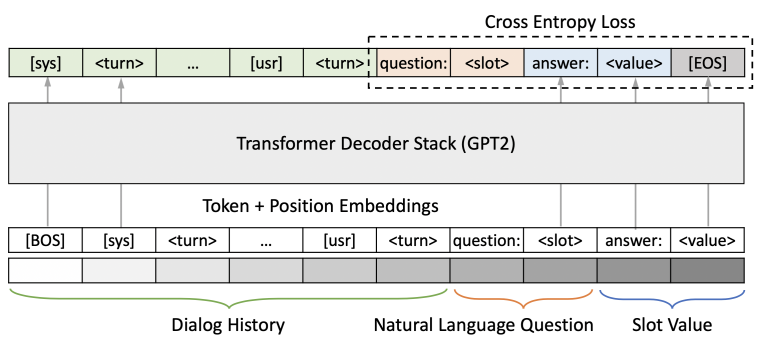

Figure 2: Our model performs DST via generative question-answering. Natural language questions for dialog slots allow our model to generalize to new slot types through its understanding of general language.

$C=\left\{y_{1}, u_{1}, \ldots y_{T}, u_{T}\right\}$. The belief state $B_{t}$ at turn $t$ comprises many tuples of slots $s \in S$ and their associated values $v_{s, t} \in V_{s}$, extracted from the conversation history $C_{t}=\left\{y_{1}, u_{1}, \ldots, y_{t}, u_{t}\right\}$. The set of possible values $V_{s}$ can be arbitrarily large (e.g. possible hotel names), so we represent these values as sequences of vocabulary tokens $v_{s, t}=\left\{w_{1}, w_{2}, \ldots, w_{k}\right\}, w_{i} \in \mathcal{W}$. At inference time we pose a natural language question $s=\left\{w_{1}, \ldots, w_{n}\right\}$ and our model predicts an answer (slot value $v_{s, t}$ ) based on its understanding of the dialog history $C_{t}$. To predict the belief state $B_{t}$, our model independently answers $|S|$ different questions (Figure 1). In zero-shot DST, the system must predict values for slots outside of the initial ontology - these slot queries correspond to arbitrary natural language questions $s^{\prime}$ about entities and relationships in the conversation $C_{t}$.

Generalizing to New Domains and Slots Dialog State Tracking systems in real-world settings must scale to new users and services, accommodating new slot values (e.g. a new movie release) as well as new domains and slot types (e.g. a service update, or a new connected API). Existing methods require the developer to either write a complete ontology of slots and allowed values or modify their model architecture to add slot-specific prediction heads (Chen et al., 2020). Span-based approaches (Zhang et al., 2019; Zhou and Small, 2019) can correctly predict values that appear verbatim in a conversation but fail when a user paraphrases or mis-phrases a value. They also fall back to treating open-valued slots as classification problems (Zhang et al., 2019; Gao et al., 2020). We approach DST as an ontology-free generative question answering task, as generative methods (Wu et al., 2019; Kumar et al., 2020) have shown promise in few-shot and supervised DST settings. 


\begin{tabular}{lrr}
\hline & JGA (\%) & \# Params \\
\hline DistilGPT2 LM & 36.35 & $82 \mathrm{M}$ \\
DistilGPT2 CLM no PT & 39.34 & \\
DistilGPT2 CLM & 49.55 & \\
$\quad$ +Question (CLMQ) & $\underline{50.83}$ & \\
\hline GPT2 CLMQ & 51.02 & $124 \mathrm{M}$ \\
\hline GPT2-medium CLMQ & $\mathbf{5 2 . 5 8}$ & $355 \mathrm{M}$ \\
\hline
\end{tabular}

Table 1: Ablation study of our framework, reporting supervised JGA on the MultiWOZ 2.1 test set.

While some approaches toward DST as QA learn a set of embeddings for each slot and/or domain (Gao et al., 2019; Wu et al., 2019; Kumar et al., 2020), this is not robust to unseen slots. We encode slots as natural language questions-manually formulating one question per slot-allowing us to share a pre-trained encoder for both dialog context and slot to leverage shared linguistic knowledge (Gao et al., 2020). Thus, our model is also agnostic to ontologies and can answer arbitrary English questions about the dialog history. We treat DST via $\mathrm{QA}$ as a conditional language modeling task, and train our model to predict the conditional likelihood of question (slot $s$ ) and answer (value $v_{s, t}$ ) tokens given a dialog context $C_{t}$ at a given turn $t$ :

$$
P\left(v_{s, t}, s \mid C_{t}\right)=P\left(v_{s, t} \mid s, C_{t}\right) * P\left(s \mid C_{t}\right)
$$

At inference time, the model is given the dialog context alongside a question- $\left[C_{t} ; s\right]$ —and asked to predict the value $v_{s, t}$ for that slot.

\section{Model Architecture}

For our conditional language model, we compared two common architectures: 1) an encoder-decoder model (Sutskever et al., 2014) with a bi-directional encoder; and 2) a purely auto-regressive decoderonly model. We conducted preliminary experiments using both a Transformer (Vaswani et al., 2017) encoder-decoder language model pre-trained using a de-noising auto-encoder objective (Lewis et al., 2020), as well as a Transformer decoder pre-trained with next-token prediction on English web pages. We achieved $1 \%$ better supervised DST performance with the decoder-only model in half the training time. Our model architecture thus comprises a Transformer decoder language model that allows us to leverage pre-trained language models like GPT2 (Radford et al., 2019) and common-sense world knowledge accrued through pre-training (Petroni et al., 2019).

\begin{tabular}{lrr}
\hline & MultiWOZ & DSTC8 \\
\hline Train & 7,906 & 16,142 \\
Validation & 1,000 & 2,482 \\
Test & 1,000 & 4,201 \\
\hline Domains & 5 & 19 \\
\hline Slots & 30 & 124 \\
Open & 9 & 59 \\
Numeric & 5 & 12 \\
Temporal & 5 & 10 \\
Categorical & 11 & 43 \\
\hline
\end{tabular}

Table 2: Dataset statistics for MultiWOZ 2.1 and DSTC8: number of dialogs in each split, number of domains, and slots with slot category breakdowns.

We use a BPE (Sennrich et al., 2016) tokenizer to convert input text into a sequence of tokens. These are embedded in $\mathbb{R}^{h}$ and added to an $\mathbb{R}^{h}$ sinusoidal positional embedding. This input embedding is processed by $l$ Transformer layers with hidden dimensionality $h$, each of which applies multi-headed attention with $k$ heads followed by a feed-forward layer with a softmax nonlinearity. The final output hidden states are then projected into our vocabulary space of 50,257 sub-word tokens. We initialize our model weights with DistilGPT2 (Sanh et al., 2019), GPT2 (Radford et al., 2019), or GPT2-medium with $h=768,768,1024$, $l=6,12,24$, and $k=12,12,16$ respectively.

As seen in Figure 2, our input sequence consists of a concatenation of dialog context $C_{t}$, slot query $s$, and slot value $v_{s, t}:\left[C_{t} ; s ; v_{s, t}\right]$. We prepend each utterance with a speaker token [usr] or [sys] for a user or system speaker to allow our model to identify additional context about each utterance. We pre-pend the slot query and value with question: and answer: respectively to distinguish slot queries from user-posed questions in the conversation. At training time, we calculate a cross-entropy loss similar to encoder-decoder models by maximizing the log likelihood of the slot query and value conditioned on the dialog context:

$$
P\left(s, v_{s, t} \mid C_{t}\right)=\prod_{i}^{n} P\left(x_{i} \mid x_{<i}, C_{t}\right)
$$

where $n=\left|\left[s ; v_{s, t}\right]\right|$. We find through ablation experiments on our architecture that this loss computation method out-performs a naïve languagemodeling approach that maximizes log likelihood of the full concatenated sequence $\left[C_{t} ; s ; v_{s, t}\right]$ via the factorized joint distribution (Peng et al., 2020; 


\begin{tabular}{lccc}
\hline Model & Type & JGA & NLQ \\
\hline TRADE (Wu et al., 2019) & G & 45.60 & \\
SUMBT (Lee et al., 2019)* & C & 46.70 & \\
STARC (Gao et al., 2020)* & C+S & 49.48 & Y \\
MA-DST (Kumar et al., 2020) & G & 51.88 & \\
GPT2-m CLMQ & G & $\mathbf{5 2 . 5 8}$ & Y \\
\hline \hline
\end{tabular}

Table 3: Supervised DST performance on MultiWOZ 2.1 of our model (underlined) compared to prior methods capable of zero-shot inference. Models using natural language questions (NLQ) are marked. *Requires access to slot-value ontologies at inference time.

Hosseini-Asl et al., 2020):

$$
P(x)=\prod_{i}^{n} P\left(x_{i} \mid x_{<i}\right)
$$

This allows for flexibility in learned representations for dialog context while regularizing slot query hidden states.

\section{Data}

We perform our experiments on MultiWOZ (Budzianowski et al., 2018), which contains over $10 \mathrm{~K}$ single- and multi-domain task-oriented dialogs written by crowd-workers. We use the 2.1 version, with corrected and standardized annotations from Eric et al. (2020). We follow Wu et al. (2019) in lower-casing all dialogs and removing dialogs from training-only domains (Police and Hospital). The final dataset contains 9,906 conversations from 5 domains (Restaurant, Hotel, Attraction, Train, Taxi) covering 30 domain-slot pairs. Each dialog contains an average of 7 user and system turns.

We also experiment with augmenting our training dataset in zero-shot settings with observations drawn from the DSTC8 (Kim et al., 2019) dataset, ${ }^{1}$ which contains 16,152 dialogs from 45 domains. DSTC8 was created via template-based dialog models provided with service APIs, and then edited by crowd-workers (Shah et al., 2018). We normalize domains and slots corresponding to the same domain (e.g. Bus_1, Bus_2) for a total of 19 domains and 124 slot types in DSTC8. We further manually annotate each dataset with slot value types: open-valued (e.g. Hotel Name), numeric (e.g. Restaurant Guests), temporal (e.g. Taxi LeaveAt), and categorical (e.g. Attraction Type). Dataset statistics are shown in Table 2.

\footnotetext{
${ }^{1}$ https://github.com/

google-research-datasets/ dstc8-schema-guided-dialogue
}

\begin{tabular}{lccl}
\hline Model & Type & JGA & Extra Supervision \\
\hline DSTQA & C+S & 51.17 & Knowledge Graph \\
DS-DST & C+S & 51.21 & \\
GPT2-m CLMQ & G & 52.58 & \\
SOM-DST & G & 53.68 & Previous Dialog State \\
SST & C & 55.23 & Schema \\
TripPy & S & 55.30 & Previous Dialog Actions \\
SimpleToD & G & $\mathbf{5 5 . 7 2}$ & Actions (Training) \\
\hline
\end{tabular}

Table 4: Supervised DST performance on MultiWOZ 2.1 of our model (underlined) against state-of-the-art DST methods incapable of zero-shot inference.

\section{Experiments}

We measure DST performance via Joint Goal Accuracy (JGA): the proportion of turns with all belief slots predicted correctly, including those not present. In Section 5.1, we evaluate our model on fully supervised DST, in which all domains and slots are known at training time. In Section 5.2, we investigate zero-shot domain adaptation in which the model is evaluated on conversations from an unseen domain with previously unseen slots. We then explore how our framework seamlessly accommodates teaching a model to predict slot carryover (Section 5.3) and transfer learning with significantly more diverse domains and slot types (Section 5.4). To measure zero-shot JGA, we follow Campagna et al. (2020) and only consider slots specific to the held-out domain. We focus our analysis on the zero-shot setting, as our goal is to build DST systems that can easily and effectively generalize to new domains and services. We train all models to convergence with a maximum of 10 epochs on Nvidia V100 GPUs, using the Lamb optimizer (You et al., 2020) with a base learning rate of 2e-5. All predictions are made using greedy decoding.

\subsection{Supervised DST}

We first evaluate on the commonly benchmarked supervised DST task to demonstrate performance competitive with state-of-the-art. In this setting we compare our approach against prior methods capable of zero-shot inference in Table 3-TRADE, STARC, SUMBT, and MA-DST-and those incapable of doing so in Table 4, including DSTQA (Zhou and Small, 2019), DS-DST (Zhang et al., 2019), SOM-DST (Kim et al., 2020), SST (Chen et al., 2020), TripPy (Heck et al., 2020), and SimpleToD (Hosseini-Asl et al., 2020). Our model outperforms all prior models that support zeroshot generalization and is competitive with meth- 


\begin{tabular}{lrrrrr}
\hline & Rest. & Hot. & Attr. & Train & Taxi \\
\hline TRADE & 12.59 & 14.20 & 20.06 & 22.39 & 59.21 \\
MA-DST & 13.56 & 16.28 & 22.46 & $\underline{22.76}$ & 59.27 \\
SUMBT & $\underline{16.50}$ & $\underline{19.80}$ & $\underline{22.60}$ & 22.50 & $\underline{59.50}$ \\
\hline Ours (GPT2) & 21.05 & 18.54 & 23.67 & 24.34 & 59.10 \\
Ours (GPT2-m) & $\mathbf{2 6 . 1 7}$ & $\mathbf{2 4 . 4 1}$ & $\mathbf{3 1 . 3 1}$ & $\mathbf{2 9 . 0 7}$ & $\mathbf{5 9 . 6 1}$ \\
\hline
\end{tabular}

Table 5: Zero-shot domain adaptation JGA (\%) on MultiWOZ 2.1 test set for recent works and our models with question loss, on the (Rest)aurant, (Hot)el, (Attr)action, Train, and Taxi domains. Previous stateof-the-art results are underlined, with new best bolded.

ods that focus solely on supervised DST-most of which require extra supervision at training and inference time, including dialog actions and prior dialog states. We distinguish models by their prediction type as (C)lassification-, (S)pan extraction-, and $(\mathrm{G})$ eneration-based methods.

As seen in Table 1, our formulation of DST as a generative QA task benefits significantly from the usage of a conditional decoder-style model. A standard auto-regressive language modeling formulation (LM) with loss computed over the entire input sequence achieves 13\% lower JGA compared to computing cross entropy loss only over slot value tokens (CLM). Pre-training is also crucialwe see a 10-point drop in JGA when randomly initializing model weights (no PT) compared to initializing from pre-trained DistilGPT2 weights. We also compare two other sizes of our models: GPT2-based-comparable in size to SUMBT's (Lee et al., 2019) 112M parameters-and GPT2medium-based - comparable in size to STARC's (Gao et al., 2020) 355M parameters. We find that scaling the size of our model results in modest improvements in supervised JGA. We hypothesize that extending our loss to cover both slot query and value tokens (+Question/CLMQ) helps regularize the hidden representations of question tokens, and we achieve a $1.3 \%$ improvement in JGA.

\subsection{Zero-Shot DST}

Our primary focus lies in the zero-shot domain adaptation setting, where conversations and target slots at inference time come from unseen domains. We use a leave-one-out setup, training our models on four domains from MultiWOZ and evaluating on the held-out domain. Our model must understand a wide variety of possible questions about unseen conversations to generalize well. We compare our model against strong baseline models for zero-shot DST: TRADE, SUMBT, and MA-DST; Table 5 contains results from our models alongside baseline results reported by Kumar et al. (2020) and Campagna et al. (2020). These models represent slots as domain-slot tuples: TRADE learns a separate embedding for each domain and word in slot names, while SUMBT and MA-DST encode domain-slot tuples via BERT (Devlin et al., 2019) and an RNN encoder, respectively.

Our GPT2-medium based model achieves stateof-the-art zero-shot performance on all five domains, and by a significant (5-10\%) margin on the Restaurant, Hotel, Attraction, and Train domains. While increased model size modestly impacts supervised DST performance (Table 1), larger models perform significantly better in a zero-shot setting with average absolute gains of $4.8 \%$ and relative gains of $22 \%$ in JGA across domains. Such improvements are consistent with findings from Brown et al. (2020) that up-sizing language models improves zero-shot performance across various tasks and Petroni et al. (2019), who observe that larger pre-trained models can retain more commonsense and world knowledge from their pre-training corpus-which may help our model understand queries for unseen domains and slots.

Effect of Natural Language Questions Prior work that frames DST as QA typically represents the slot query as a concatenation (tuple) of domain and slot name. Zhang et al. (2019) explore the impact of three different slot representationsdomain-slot tuples, short slot descriptions, and full questions-on a hybrid classification-extraction model for DST, and find little difference in performance. However, we find that full questions work much better than domain-slot tuples for our generative framework, especially in zero-shot DST. We hypothesize that natural language questionsstructurally similar to dialog utterances and pretraining sentences - allow our model to best leverage its linguistic knowledge with minimal friction when jointly encoding the dialog history, slot query, and slot value.

Wu et al. (2019) find that zero-shot generalization in models that represent slots as tuples is primarily due to shared slot names between domains (e.g. Taxi and Train 'leaveAt'). In a real-world setting a newly added dialog service is unlikely to share slot names verbatim with existing services. To fairly compare tuples and natural language questions under our framework, we per- 


\begin{tabular}{|c|c|c|c|c|c|c|c|}
\hline USER & \multicolumn{7}{|c|}{ My friend told me about Carolina Bed and Breakfast. Do you know anything about it? } \\
\hline SYS & \multicolumn{7}{|c|}{ It's a 4 star guesthouse. What would you like to know about it? } \\
\hline USER & \multicolumn{7}{|c|}{ Can you give me the postcode? And, do they have internet? } \\
\hline SYS & \multicolumn{7}{|c|}{ The postcode is cb13nx; they have internet. } \\
\hline USER & \multicolumn{7}{|c|}{ Thanks. Any boat attractions in the west? } \\
\hline SYS & \multicolumn{7}{|c|}{ Nothing in west. Closest boat is the Cambridge Punter in centre. Too far? } \\
\hline USER & \multicolumn{7}{|c|}{ Yes, it is. How about a museum? } \\
\hline Error Modality & Slot & Gold & Prediction & Open & Numeric & Temporal & Categorical \\
\hline Spurious & (Attraction, Name) & $\mathrm{n} / \mathrm{a}$ & cambridge punter & $8.4 \%$ & $22.3 \%$ & $47.7 \%$ & $16.0 \%$ \\
\hline Ignored & (Hotel, Internet) & yes & $\mathrm{n} / \mathrm{a}$ & $65.3 \%$ & $53.5 \%$ & $19.9 \%$ & $76.8 \%$ \\
\hline Wrong Value & (Attraction, Type) & museum & boat & $26.3 \%$ & $24.2 \%$ & $32.4 \%$ & $7.2 \%$ \\
\hline
\end{tabular}

Table 6: Example of different classes of DST errors, and the proportion of errors they make up across the four slot categories for all five domains in a zero-shot setting. Latest (target) turn is bolded.

form zero-shot experiments using each representation. For tuple-based questions, our model takes as slot query a synonym of the slot name (e.g. Taxi 'leaveAt' $\rightarrow$ 'Pick Up Time') instead of a full question (e.g. 'What time does the user want the taxi to pick them up $i$ ). Full question models achieved $6 \%$ higher per-domain JGA compared to slot-tuple models, supporting the notion that slot-tuple models memorize slot names rather than understanding their meaning and thus do not generalize well in real-world settings. Using full questions, our model (Table 5) achieves state-of-the-art performance in zero-shot settings.

Error Modalities To analyze our model, we follow Gao et al. (2020) and categorize DST errors in three modalities: 1) the model predicts a spurious value for an irrelevant slot; 2) the model ignores a relevant slot; and 3) the model correctly infers the presence of a slot but predicts a wrong value. Table 6 shows examples of each type of error for a sample conversation, and what proportion of errors they make up in each slot category for our GPT2m CLMQ model in a zero-shot setting. Temporal slots are least likely to be ignored by our model, as verbatim $\mathrm{HH}: \mathrm{MM}$ values are easily identifiable in a conversation. However, it is difficult to distinguish between closely related unseen temporal slots like 'leaveAt' and 'arriveBy'. Values for categorical, numeric, and open-valued slots on the other hand can comprise common (non-slot) phrases used in conversation, and thus it is easy for our model to ignore such slot references.

We also examine the source of dialog slots: users explicitly express the majority (79.5\%) of slot values, while a minority are either derived via user reactions to system suggestions $(9.7 \%)$ or implicitly valued $(10.8 \%)$-not present verbatim in a conversation. However, our errors are distributed evenly between user, system, and implicit sourced slotssuggesting that it is challenging for our model to track dialog states that are updated reactively via user feedback. We thus see a future opportunity to improve DST models by emphasizing multi-hop reasoning and common-sense inference.

\subsection{Predicting Carried Over Slots}

Long-range dependencies and slot values carried over from early turns are particularly important to model for accurate DST in long conversations (Kumar et al., 2020). We observe this in the zero-shot setting: our model is able to predict all slots accurately for $61 \%$ of conversation first-turns, dropping to $46 \%$ after one turn, and $5.7 \%$ after seven turns (the average conversation duration). We implement an oracle module to discard predictions when a dialog state does not need updating, obtaining an upper bound for DST improvements due to carryover prediction. With this oracle, we see an average 5-point improvement in JGA across domain, indicating that carry-over prediction can greatly benefit our model. State-of-the-art models for fully supervised DST often rely on explicitly processing previous dialog states - via slot-value graphs (Zhou and Small, 2019; Chen et al., 2020) or as a separate input to the model at each turn (Heck et al., 2020; Kim et al., 2020). In our framework we can target slot carry-over by training a model to predict a carried over token in place of the true slot value whenever a slot value does not need updating at the current turn (+ Carryover). At inference time, we replace predicted carry-over tokens with the slot's last predicted value.

Our carry-over implementation improved JGA for all domains (Table 7) by an average of 3.14\%, and improved JGA across all context lengthswith the largest improvements $(+7 \%)$ at the sec- 


\begin{tabular}{lrrrrr}
\hline & Rest. & Hotel & Attr. & Train & Taxi \\
\hline Previous SOTA & 16.50 & 19.80 & 22.60 & 22.76 & 59.50 \\
\hline GPT2 CLMQ & 21.05 & 18.54 & 23.67 & 24.34 & 59.10 \\
+ Carryover & 24.00 & 19.91 & 28.45 & 30.75 & 59.29 \\
+ DSTC8 & $\underline{24.65}$ & $\underline{22.94}$ & $\underline{34.30}$ & $\underline{38.55}$ & $\underline{59.68}$ \\
\hline GPT2-m CLMQ & 26.17 & 24.41 & 31.31 & 29.07 & 59.68 \\
+ CO, DSTC8 & $\mathbf{2 7 . 6 9}$ & $\mathbf{2 4 . 8 8}$ & $\mathbf{4 2 . 3 9}$ & $\mathbf{4 1 . 0 5}$ & $\mathbf{6 0 . 3 2}$ \\
\hline
\end{tabular}

Table 7: Zero-shot JGA on MultiWOZ 2.1 test set with carry-over prediction and transfer learning.

ond and third turn of a conversation (Figure 4). The carried over token allows our model to hedge against low confidence slots, falling back to predictions from previous turns where the target slot may be directly mentioned. This helps reduce the wrong value error rate by an average of $31 \%$ across each domain. Our model can also propagate null values with carry-over, reducing spurious predictions by an average of $36 \%$ across domains. However, we also observe our carry-over model propagating $78 \%$ of its errors from previous turns, suggesting that further improvements can result via accurately predicting slot updates.

\subsection{Transfer Learning for Generalization}

Our framework is ontology-agnostic and thus easily supports transfer learning without modifying the architecture by simply writing natural language questions for additional slots. Gao et al. (2020) found that intermediate fine-tuning of RoBERTaLarge (Liu et al., 2019) on passage-based QA tasks (Fisch et al., 2019) improved zero-shot DST performance. In preliminary experiments, we found no significant impact from intermediate fine-tuning on the SQuAD v2.0 (Rajpurkar et al., 2018) passagebased QA dataset. However, we observe significant improvements when training with joint, noncurriculum learning (McCann et al., 2018; Raffel et al., 2020)_augmenting our training data with an equal number of examples sampled from DSTC8, taking care to remove data from the held-out domain in both MultiWOZ and DSTC8.

Our framework allows for easy joint optimization with carry-over and transfer learning: by training new models on MultiWOZ 2.1 augmented with DSTC8 (+ DSTC8) we gain a further average 3.5point improvement in per-domain JGA (Table 7). On average, our model makes $29 \%$ fewer spurious errors, and $6.9 \%$ fewer errors in open-valued slots, suggesting that our model scales well with additional training data with semantically distinct

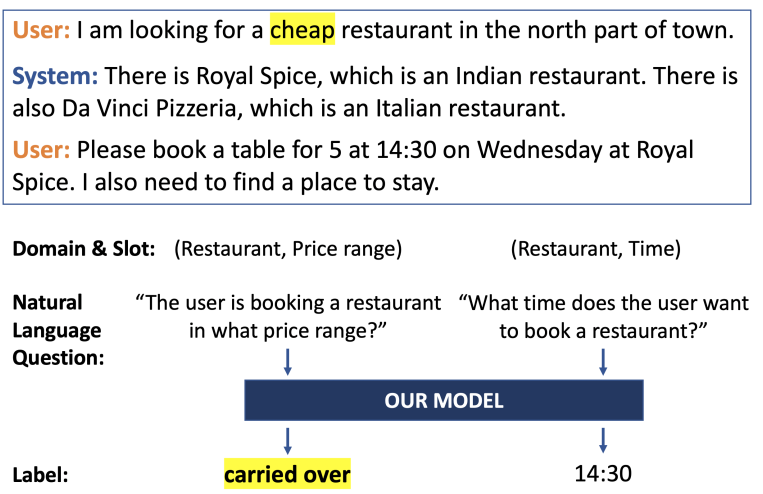

Figure 3: Our model can be trained to predict the presence of slot carry-over by replacing slot values from previous turns with the carried over token.

slot types and values. Our model also makes 9.7\% fewer errors on categorical slots and $63 \%$ fewer mistakes where it assigns the value of one categorical slot to another, despite being unable to observe the set of possible categorical options-suggesting that exposure to more diverse categorical slots allows our model to better understand and distinguish between such slots. While temporal slots comprise only $17 \%$ of MultiWOZ and 10\% of DSTC8 slots, these additional examples seem to help our model better disambiguate temporal references and make $32 \%$ fewer errors in such slots.

By applying both carry-over and transfer learning to our largest model, we observe further improvements in zero-shot JGA for all domainsaveraging 5.1 points better than GPT2-m CLMQ, for an average gain of $11 \%$ JGA over previous state-of-the-art across domains (Table 7).

\section{Qualitative Analysis}

We manually reviewed 300 errors made by our GPT2-medium CLMQ model in the zero-shot setting - annotating 20 errors from each modality (spurious, ignored, wrong value) from each domain with the gold label quality and perceived cause of error totaling 300 annotated examples. As widely observed in recent DST work (Zhou and Small, 2019; Kumar et al., 2020), a significant proportion of DST errors on MultiWOZ are unavoidablecaused by annotation errors. While version 2.1 corrected some of these, annotation errors and inconsistencies remain responsible for $30 \%$ of sampled errors-in particular, in $10 \%$ of errors the original annotator did not record reactive preferences while in 5\% of errors the original annotator did. These inconsistencies can hurt our model's ability to infer 


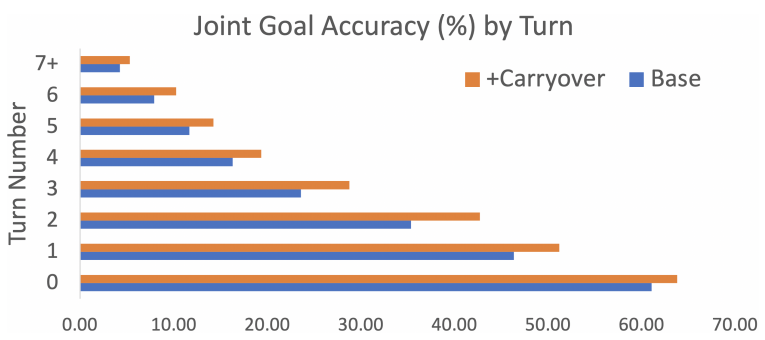

Figure 4: Per-turn JGA on MultiWOZ Test set for GPT2-CLMQ with and without carry-over prediction.

reactive and implied requirements and preferences.

We are also particularly interested in slot transfers-when our model mistakenly predicts one slot's value for a different slot, comprising $36 \%$ of our manually reviewed errors. In the Taxi and Hotel domains, our model transfers slots from the same domain over $75 \%$ of the time, with most swaps occurs between same-category slots (e.g. temporal slots like Taxi 'LeaveAt' and 'ArriveBy'). Slots in these domains are closely semantically related, with values that can fit any slot of that category (e.g. 13:10 vs. 15:15). While a human can easily infer that the earlier of two times must be departure and the later arrival, our model has no inherent understanding of temporal mechanics or numeracy (Wallace et al., 2019). In future work, we will explore learning such knowledge directly via hierarchical softmax output distributions to distinguish between output modalities (Spithourakis and Riedel, 2018), and fine-tuning our model with contrastive losses to learn to rank numerals and times (Hoffer and Ailon, 2015).

For Restaurant, Attraction, and Train, our model tends to swap slot values with those from other domains in the conversation. This is often due to semantically similar slots whose values, at first glance, may not be obviously identifiable as such (e.g. 'Bridge' or 'The Place'). Kumar et al. (2020) similarly observe a particularly high incidence of slot transfers between different-domain 'Name' slots. Other such slots include price ranges and numbers of guests. We have seen that data augmentation with DSTC8 can improve our model's ability to disambiguate such slots-this suggests that we could further improve our model by exposing it to in-domain, conversational reading comprehension data.

While no such dataset currently exists, in future work we aim to explore using question generation (Du et al., 2017) and paraphrasing (Tseng et al., 2014) models to perform in-domain data augmen- tation, creating reading comprehension questions for task-oriented dialogs that targeting entities and relations not covered by an ontology. We also wish to explore methods for generating general reading comprehension questions for out-of-domain conversations (Shakeri et al., 2020) to improve our model's domain adaptation ability.

\section{Related Works}

Modern dialog state tracking seeks to capture evolving user intents in a structured belief state (Thomson and Young, 2010). Traditional systems rely on hand-crafted features (Henderson et al., 2014) and classify slot values from a fixed ontology (Mrksic et al., 2017; Ramadan et al., 2018). Gao et al. (2019) and Zhou and Small (2019) fill some slots via spans extracted from dialog history, although they treat non-numeric slots as categorical. Generative methods (Xu and $\mathrm{Hu}, 2018$; Wu et al., 2019) can predict arbitrary unseen values, with HosseiniAsl et al. (2020) achieving state-of-the-art supervised DST performance in MultiWOZ 2.1 although they cannot predict unseen slots.

By posing DST as generative QA, our framework can leverage language models pre-trained on open-domain documents (Radford et al., 2019) to understand unfamiliar queries. Like Gao et al. (2020), we seek to answer natural language questions about each slot. We contrast our approach to zero-shot DST - which never has access to slots or dialog from the target domain - and that of Campagna et al. (2020), who expose their 'zero-shot' models to synthetic in-domain conversations that require access to the full ontology of the 'held-out' evaluation domain.

We take inspiration from previous work that frames a wide selection of natural language understanding (NLU) tasks (Wang et al., 2019) as QA (McCann et al., 2018) and span extraction (Keskar et al., 2019). While question-answering can be posed as a span extraction task (Wang et al., 2016), generative approaches have proven successful in answering questions about complex passages (Fan et al., 2019). We use a language modeling approach, taking cues from Raffel et al. (2020) who demonstrate that a large language model trained on next-token prediction can learn to solve many different NLU tasks posed as text. Recent work has also shown that large pre-trained language models can generalize to new NLU tasks with few or no examples (Brown et al., 2020), and we leverage 
this alongside world knowledge acquired during the pre-training process (Petroni et al., 2019) to build a DST model that is robust to new domains and slot-value ontologies.

\section{Conclusion}

This paper proposes a conditional language modeling approach to multi-domain DST posed as a generative question answering task. By leveraging natural language questions as state queries, our model can generalize to unseen domains, slots, and values via its understanding of language. Our model achieves state-of-the-art zero-shot results on the MultiWOZ 2.1 dataset with average per-domain absolute improvements of $5.9 \%$ joint accuracy. We also demonstrate that our framework is easily extensible to support transfer learning and learning slot carry-over. In the future, it is worth exploring mechanisms for our model to better understand relative temporal values and general reading comprehension questions from conversations in order to disambiguate semantically similar dialog slots.

\section{Acknowledgments}

We thank Ben Liu, Maryam Fazel-Zarandi, Anuj Goyal, and anonymous reviewers for providing valuable feedback on this work. Work was performed during first author's internship at Amazon Alexa AI. Findings and observations are of the authors only, and do not necessarily reflect the views of Amazon or UCSD.

\section{References}

Tom B. Brown, Benjamin Mann, Nick Ryder, Melanie Subbiah, Jared Kaplan, Prafulla Dhariwal, Arvind Neelakantan, Pranav Shyam, Girish Sastry, Amanda Askell, Sandhini Agarwal, Ariel Herbert-Voss, Gretchen Krueger, Tom Henighan, Rewon Child, Aditya Ramesh, Daniel M. Ziegler, Jeffrey Wu, Clemens Winter, Christopher Hesse, Mark Chen, Eric Sigler, Mateusz Litwin, Scott Gray, Benjamin Chess, Jack Clark, Christopher Berner, Sam McCandlish, Alec Radford, Ilya Sutskever, and Dario Amodei. 2020. Language models are few-shot learners. In Advances in Neural Information Processing Systems 33: Annual Conference on Neural Information Processing Systems 2020, NeurIPS 2020, December 6-12, 2020, virtual.

Pawel Budzianowski, Tsung-Hsien Wen, Bo-Hsiang Tseng, Iñigo Casanueva, Stefan Ultes, Osman Ramadan, and Milica Gasic. 2018. Multiwoz - A largescale multi-domain wizard-of-oz dataset for taskoriented dialogue modelling. In Proceedings of the
2018 Conference on Empirical Methods in Natural Language Processing, Brussels, Belgium, October 31 - November 4, 2018, pages 5016-5026.

Giovanni Campagna, Agata Foryciarz, Mehrad Moradshahi, and Monica S. Lam. 2020. Zero-shot transfer learning with synthesized data for multi-domain dialogue state tracking. In Proceedings of the 58th Annual Meeting of the Association for Computational Linguistics, ACL 2020, Online, July 5-10, 2020, pages $122-132$.

Lu Chen, Boer Lv, Chi Wang, Su Zhu, Bowen Tan, and Kai Yu. 2020. Schema-guided multi-domain dialogue state tracking with graph attention neural networks. In The Thirty-Fourth AAAI Conference on Artificial Intelligence, AAAI 2020, The ThirtySecond Innovative Applications of Artificial Intelligence Conference, IAAI 2020, The Tenth AAAI Symposium on Educational Advances in Artificial Intelligence, EAAI 2020, New York, NY, USA, February 7-12, 2020, pages 7521-7528.

Jacob Devlin, Ming-Wei Chang, Kenton Lee, and Kristina Toutanova. 2019. BERT: pre-training of deep bidirectional transformers for language understanding. In Proceedings of the 2019 Conference of the North American Chapter of the Association for Computational Linguistics: Human Language Technologies, NAACL-HLT 2019, Minneapolis, MN, USA, June 2-7, 2019, Volume 1 (Long and Short Papers), pages 4171-4186.

Xinya Du, Junru Shao, and Claire Cardie. 2017. Learning to ask: Neural question generation for reading comprehension. In Proceedings of the 55th Annual Meeting of the Association for Computational Linguistics, ACL 2017, Vancouver, Canada, July 30 August 4, Volume 1: Long Papers, pages 1342-1352.

Mihail Eric, Rahul Goel, Shachi Paul, Abhishek Sethi, Sanchit Agarwal, Shuyang Gao, Adarsh Kumar, Anuj Kumar Goyal, Peter Ku, and Dilek HakkaniTür. 2020. Multiwoz 2.1: A consolidated multidomain dialogue dataset with state corrections and state tracking baselines. In Proceedings of The 12th Language Resources and Evaluation Conference, LREC 2020, Marseille, France, May 11-16, 2020, pages 422-428.

Angela Fan, Yacine Jernite, Ethan Perez, David Grangier, Jason Weston, and Michael Auli. 2019. ELI5: long form question answering. In Proceedings of the 57th Conference of the Association for Computational Linguistics, ACL 2019, Florence, Italy, July 28-August 2, 2019, Volume 1: Long Papers, pages 3558-3567.

Adam Fisch, Alon Talmor, Robin Jia, Minjoon Seo, Eunsol Choi, and Danqi Chen. 2019. MRQA 2019 shared task: Evaluating generalization in reading comprehension. In Proceedings of the 2nd Workshop on Machine Reading for Question Answering, MRQA@EMNLP 2019, Hong Kong, China, November 4, 2019, pages 1-13. 
Shuyang Gao, Sanchit Agarwal, Tagyoung Chung, Di Jin, and Dilek Hakkani-Tür. 2020. From machine reading comprehension to dialogue state tracking: Bridging the gap. CoRR, abs/2004.05827.

Shuyang Gao, Abhishek Sethi, Sanchit Agarwal, Tagyoung Chung, and Dilek Hakkani-Tür. 2019. Dialog state tracking: A neural reading comprehension approach. In Proceedings of the 20th Annual SIGdial Meeting on Discourse and Dialogue, SIGdial 2019, Stockholm, Sweden, September 11-13, 2019, pages 264-273.

Michael Heck, Carel van Niekerk, Nurul Lubis, Christian Geishauser, Hsien-Chin Lin, Marco Moresi, and Milica Gasic. 2020. Trippy: A triple copy strategy for value independent neural dialog state tracking. In Proceedings of the 21th Annual Meeting of the Special Interest Group on Discourse and Dialogue, SIGdial 2020, 1st virtual meeting, July 1-3, 2020, pages 35-44.

Matthew Henderson, Blaise Thomson, and Steve J. Young. 2014. Word-based dialog state tracking with recurrent neural networks. In Proceedings of the SIGDIAL 2014 Conference, The 15th Annual Meeting of the Special Interest Group on Discourse and Dialogue, 18-20 June 2014, Philadelphia, PA, USA, pages 292-299.

Elad Hoffer and Nir Ailon. 2015. Deep metric learning using triplet network. In 3rd International Conference on Learning Representations, ICLR 2015, San Diego, CA, USA, May 7-9, 2015, Workshop Track Proceedings.

Ehsan Hosseini-Asl, Bryan McCann, Chien-Sheng Wu, Semih Yavuz, and Richard Socher. 2020. A simple language model for task-oriented dialogue. In Advances in Neural Information Processing Systems 33: Annual Conference on Neural Information Processing Systems 2020, NeurIPS 2020, December 612, 2020, virtual.

Nitish Shirish Keskar, Bryan McCann, Caiming Xiong, and Richard Socher. 2019. Unifying question answering and text classification via span extraction. CoRR, abs/1904.09286.

Seokhwan Kim, Michel Galley, R. Chulaka Gunasekara, Sungiin Lee, Adam Atkinson, Baolin Peng, Hannes Schulz, Jianfeng Gao, Jinchao Li, Mahmoud Adada, Minlie Huang, Luis A. Lastras, Jonathan K. Kummerfeld, Walter S. Lasecki, Chiori Hori, Anoop Cherian, Tim K. Marks, Abhinav Rastogi, Xiaoxue Zang, Srinivas Sunkara, and Raghav Gupta. 2019. The eighth dialog system technology challenge. CoRR, abs/1911.06394.

Sungdong Kim, Sohee Yang, Gyuwan Kim, and SangWoo Lee. 2020. Efficient dialogue state tracking by selectively overwriting memory. In Proceedings of the 58th Annual Meeting of the Association for Computational Linguistics, ACL 2020, Online, July 5-10, 2020, pages 567-582.
Adarsh Kumar, Peter Ku, Anuj Kumar Goyal, Angeliki Metallinou, and Dilek Hakkani-Tür. 2020. MADST: multi-attention-based scalable dialog state tracking. In The Thirty-Fourth AAAI Conference on Artificial Intelligence, AAAI 2020, The ThirtySecond Innovative Applications of Artificial Intelligence Conference, IAAI 2020, The Tenth AAAI Symposium on Educational Advances in Artificial Intelligence, EAAI 2020, New York, NY, USA, February 7-12, 2020, pages 8107-8114.

Hwaran Lee, Jinsik Lee, and Tae-Yoon Kim. 2019. SUMBT: slot-utterance matching for universal and scalable belief tracking. In Proceedings of the 57th Conference of the Association for Computational Linguistics, ACL 2019, Florence, Italy, July 28-August 2, 2019, Volume 1: Long Papers, pages 54785483.

Oliver Lemon, Kallirroi Georgila, James Henderson, and Matthew N. Stuttle. 2006. An ISU dialogue system exhibiting reinforcement learning of dialogue policies: Generic slot-filling in the TALK in-car system. In EACL 2006, 11st Conference of the European Chapter of the Association for Computational Linguistics, Proceedings of the Conference, April 37, 2006, Trento, Italy.

Mike Lewis, Yinhan Liu, Naman Goyal, Marjan Ghazvininejad, Abdelrahman Mohamed, Omer Levy, Veselin Stoyanov, and Luke Zettlemoyer. 2020. BART: denoising sequence-to-sequence pretraining for natural language generation, translation, and comprehension. In Proceedings of the 58th Annual Meeting of the Association for Computational Linguistics, ACL 2020, Online, July 5-10, 2020, pages 7871-7880.

Yinhan Liu, Myle Ott, Naman Goyal, Jingfei Du, Mandar Joshi, Danqi Chen, Omer Levy, Mike Lewis, Luke Zettlemoyer, and Veselin Stoyanov. 2019. Roberta: A robustly optimized BERT pretraining approach. CoRR, abs/1907.11692.

Bryan McCann, Nitish Shirish Keskar, Caiming Xiong, and Richard Socher. 2018. The natural language decathlon: Multitask learning as question answering. CoRR, abs/1806.08730.

Nikola Mrksic, Diarmuid Ó Séaghdha, Tsung-Hsien Wen, Blaise Thomson, and Steve J. Young. 2017. Neural belief tracker: Data-driven dialogue state tracking. In Proceedings of the 55th Annual Meeting of the Association for Computational Linguistics, ACL 2017, Vancouver, Canada, July 30 - August 4, Volume 1: Long Papers, pages 1777-1788.

Baolin Peng, Chunyuan Li, Jinchao Li, Shahin Shayandeh, Lars Liden, and Jianfeng Gao. 2020. SOLOIST: few-shot task-oriented dialog with A single pre-trained auto-regressive model. CoRR, abs/2005.05298.

Fabio Petroni, Tim Rocktäschel, Sebastian Riedel, Patrick S. H. Lewis, Anton Bakhtin, Yuxiang Wu, 
and Alexander H. Miller. 2019. Language models as knowledge bases? In Proceedings of the 2019 Conference on Empirical Methods in Natural Language Processing and the 9th International Joint Conference on Natural Language Processing, EMNLP-IJCNLP 2019, Hong Kong, China, November 3-7, 2019, pages 2463-2473.

Alec Radford, Jeffrey Wu, Dario Amodei, Daniela Amodei, Jack Clark, Miles Brundage, and Ilya Sutskever. 2019. Better language models and their implications. OpenAI Blog.

Colin Raffel, Noam Shazeer, Adam Roberts, Katherine Lee, Sharan Narang, Michael Matena, Yanqi Zhou, Wei Li, and Peter J. Liu. 2020. Exploring the limits of transfer learning with a unified text-to-text transformer. J. Mach. Learn. Res., 21:140:1-140:67.

Pranav Rajpurkar, Robin Jia, and Percy Liang. 2018. Know what you don't know: Unanswerable questions for squad. In Proceedings of the 56th Annual Meeting of the Association for Computational Linguistics, ACL 2018, Melbourne, Australia, July 1520, 2018, Volume 2: Short Papers, pages 784-789.

Osman Ramadan, Pawel Budzianowski, and Milica Gasic. 2018. Large-scale multi-domain belief tracking with knowledge sharing. In Proceedings of the 56th Annual Meeting of the Association for Computational Linguistics, ACL 2018, Melbourne, Australia, July 15-20, 2018, Volume 2: Short Papers, pages $432-437$.

Victor Sanh, Lysandre Debut, Julien Chaumond, and Thomas Wolf. 2019. Distilbert, a distilled version of BERT: smaller, faster, cheaper and lighter. CoRR, abs/1910.01108.

Rico Sennrich, Barry Haddow, and Alexandra Birch. 2016. Neural machine translation of rare words with subword units. In Proceedings of the 54th Annual Meeting of the Association for Computational Linguistics, ACL 2016, August 7-12, 2016, Berlin, Germany, Volume 1: Long Papers.

Pararth Shah, Dilek Hakkani-Tür, Gökhan Tür, Abhinav Rastogi, Ankur Bapna, Neha Nayak, and Larry P. Heck. 2018. Building a conversational agent overnight with dialogue self-play. CoRR, abs/1801.04871.

Siamak Shakeri, Cícero Nogueira dos Santos, Henghui Zhu, Patrick Ng, Feng Nan, Zhiguo Wang, Ramesh Nallapati, and Bing Xiang. 2020. End-to-end synthetic data generation for domain adaptation of question answering systems. In Proceedings of the 2020 Conference on Empirical Methods in Natural Language Processing, EMNLP 2020, Online, November 16-20, 2020, pages 5445-5460.

Georgios P. Spithourakis and Sebastian Riedel. 2018. Numeracy for language models: Evaluating and improving their ability to predict numbers. In Proceedings of the 56th Annual Meeting of the Associa- tion for Computational Linguistics, ACL 2018, Melbourne, Australia, July 15-20, 2018, Volume 1: Long Papers, pages 2104-2115.

Ilya Sutskever, Oriol Vinyals, and Quoc V. Le. 2014. Sequence to sequence learning with neural networks. In Advances in Neural Information Processing Systems 27: Annual Conference on Neural Information Processing Systems 2014, December 8-13 2014, Montreal, Quebec, Canada, pages 3104-3112.

Blaise Thomson and Steve J. Young. 2010. Bayesian update of dialogue state: A POMDP framework for spoken dialogue systems. Comput. Speech Lang., 24(4):562-588.

Ya-Min Tseng, Yi-Ting Huang, Meng Chang Chen, and Yeali S. Sun. 2014. Generating comprehension questions using paraphrase. In Technologies and Applications of Artificial Intelligence, 19th International Conference, TAAI 2014, Taipei, Taiwan, November 21-23, 2014. Proceedings, pages 310 321.

Ashish Vaswani, Noam Shazeer, Niki Parmar, Jakob Uszkoreit, Llion Jones, Aidan N. Gomez, Lukasz Kaiser, and Illia Polosukhin. 2017. Attention is all you need. In Advances in Neural Information Processing Systems 30: Annual Conference on Neural Information Processing Systems 2017, December 49, 2017, Long Beach, CA, USA, pages 5998-6008.

Eric Wallace, Yizhong Wang, Sujian Li, Sameer Singh, and Matt Gardner. 2019. Do NLP models know numbers? probing numeracy in embeddings. In Proceedings of the 2019 Conference on Empirical Methods in Natural Language Processing and the 9th International Joint Conference on Natural Language Processing, EMNLP-IJCNLP 2019, Hong Kong, China, November 3-7, 2019, pages 53065314.

Alex Wang, Amanpreet Singh, Julian Michael, Felix Hill, Omer Levy, and Samuel R. Bowman. 2019. GLUE: A multi-task benchmark and analysis platform for natural language understanding. In 7 th International Conference on Learning Representations, ICLR 2019, New Orleans, LA, USA, May 6-9, 2019.

Bingning Wang, Kang Liu, and Jun Zhao. 2016. Inner attention based recurrent neural networks for answer selection. In Proceedings of the 54th Annual Meeting of the Association for Computational Linguistics, ACL 2016, August 7-12, 2016, Berlin, Germany, Volume 1: Long Papers.

Zhuoran Wang and Oliver Lemon. 2013. A simple and generic belief tracking mechanism for the dialog state tracking challenge: On the believability of observed information. In Proceedings of the SIGDIAL 2013 Conference, The 14th Annual Meeting of the Special Interest Group on Discourse and Dialogue, 22-24 August 2013, SUPELEC, Metz, France, pages 423-432. 
Chien-Sheng Wu, Andrea Madotto, Ehsan HosseiniAsl, Caiming Xiong, Richard Socher, and Pascale Fung. 2019. Transferable multi-domain state generator for task-oriented dialogue systems. In Proceedings of the 57th Conference of the Association for Computational Linguistics, ACL 2019, Florence, Italy, July 28-August 2, 2019, Volume 1: Long Papers, pages $808-819$.

Puyang Xu and Qi Hu. 2018. An end-to-end approach for handling unknown slot values in dialogue state tracking. In Proceedings of the 56th Annual Meeting of the Association for Computational Linguistics, ACL 2018, Melbourne, Australia, July 15-20, 2018, Volume 1: Long Papers, pages 1448-1457.

Yang You, Jing Li, Sashank J. Reddi, Jonathan Hseu, Sanjiv Kumar, Srinadh Bhojanapalli, Xiaodan Song, James Demmel, Kurt Keutzer, and Cho-Jui Hsieh. 2020. Large batch optimization for deep learning: Training BERT in 76 minutes. In 8th International Conference on Learning Representations, ICLR 2020, Addis Ababa, Ethiopia, April 26-30, 2020 .

Jianguo Zhang, Kazuma Hashimoto, Chien-Sheng Wu, Yao Wan, Philip S. Yu, Richard Socher, and Caiming Xiong. 2019. Find or classify? dual strategy for slot-value predictions on multi-domain dialog state tracking. CoRR, abs/1910.03544.

Li Zhou and Kevin Small. 2019. Multi-domain dialogue state tracking as dynamic knowledge graph enhanced question answering. CoRR, abs/1911.06192. 